

Cite this: RSC Adv., 2014, 4, 34474

Received 23rd June 2014 Accepted 29th July 2014

DOI: 10.1039/c4ra06131d

www.rsc.org/advances

\section{Epitaxial graphene as an electrode material: a transistor testbed for organic and all-carbon semiconductors}

\begin{abstract}
E. Bayaya, D. Waldmann, M. Krieger and H. B. Weber*
We present a transistor testbed for novel organic and all-carbon electronic materials. Epitaxial graphene on silicon carbide $(\mathrm{SiC})$ is used as source and drain electrodes. The gate is implemented as a bottom gate. Due to Fermi level pinning at the graphene/SiC interface, the gate is effective in the channel area only. The semiconductor above source and drain contacts and the graphene itself are unaffected by the bottom gate. This leads to a clear distinction of electronic properties in the sharply separated channel and contact areas. As an example, we investigate $\mathrm{P} 3 \mathrm{HT}$ and fullerene films, representing standard p-type and n-type semiconducting materials. In particular, for P3HT an ohmic contact to graphene was observed, and an accurate and consistent determination of transistor parameters was achieved. The fullerene transistor showed a high on/off ratio of $3 \times 10^{3}$. The testbed offers the opportunity to determine semiconductor parameters of novel materials under very well-defined conditions.
\end{abstract}

\section{Introduction}

Graphene is an interesting electronic material with ultra-high charge carrier mobilities. ${ }^{1}$ Therefore, it has been proposed as a transistor material with a very fast response., ${ }^{2,3}$ Due to the lack of a gap in its energy spectrum, however, its switching capabilities are poor. ${ }^{4}$ In this paper, we consider graphene as a metal, suited for atomically flat electrodes. The following experiment proposes a transistor testbed that can be used to evaluate any semiconducting material on graphene electrodes. Graphene has previously been used in several experiments as a contact material to organic semiconductors ${ }^{5-10}$ and to conventional semiconductors. ${ }^{\mathbf{1 1}}$ In our case, the graphene has epitaxial quality, which brings along a well-defined work function. For the proof of principle, we use standard carbon-based semiconductors in the transistor channel: P3HT as one of the most frequently used p-type polymer semiconductors ${ }^{12}$ and fullerenes as an example for n-type semiconductors. ${ }^{13,14}$ This material combination has the interesting property that the all-important metal/semiconductor interface is entirely carbon based. Further, this transistor concept avoids any roughness in the electrode as well as in the channel area, as the semiconductor spreads over the atomically flat graphene electrodes and the atomically flat $\mathrm{SiC}$ substrate without a significant step in between.

The graphene species we use is epitaxial graphene on a $\mathrm{SiC}$ (0001) wafer, grown by thermal decomposition. ${ }^{15}$ It uses $\mathrm{SiC}$ both as a substrate but also as carbon source for the graphene

Lehrstuhl für Angewandte Physik, Friedrich-Alexander-Universität Erlangen-Nürnberg, Staudtstr. 7, 91058 Erlangen, Germany.E-mail: heiko.weber@fau.de growth. The result is large-area graphene in epitaxial registry with respect to the single-crystal wafer. As SiC is a wide-bandgap semiconductor, it can be chosen as highly insulating, but also as a well conducting material, depending on doping. In our case, we have created a conducting plane $\sim 1200 \mathrm{~nm}$ below the surface of a semi-insulating $6 \mathrm{H}-\mathrm{SiC}$ wafer by implantation of dopants. ${ }^{16,17}$ This serves as electrostatic gate with respect to the graphene plane at the wafer surface, because the SiC area in between acts as gate dielectric up to applied voltages of $\sim 100 \mathrm{~V}$.

\section{Experimental}

The bottom gate was structured and contacted in a semiinsulating $6 \mathrm{H}-\mathrm{SiC}(0001)$ wafer (II-VI Inc.) either by aluminum implantation $\left([\mathrm{Al}]=2.13 \times 10^{13} \mathrm{~cm}^{-2}\right.$, used for P3HT transistor in Fig. 3), or by nitrogen implantation with reduced ion dose $\left([\mathrm{N}]=1.1 \times 10^{13} \mathrm{~cm}^{-2}\right.$, used for fullerene and P3HT transistors), following the strategies and recipes of ref. 16 and 17. Subsequently, the samples were heated in $\mathrm{Ar}$ atmosphere at $1750{ }^{\circ} \mathrm{C}$ in a cold-wall reactor. Under these conditions, highquality monolayer graphene (MLG) is formed. ${ }^{15}$ It is known that this graphene species is not charge neutral, but has a charge carrier density of $n=10^{13} \mathrm{~cm}^{-2}$, a work function of $\Phi=$ $4.31 \mathrm{eV}$, and charge carrier mobilities of $\mu_{\text {graphene }}=1000 \mathrm{~cm}^{2}$ $\mathrm{V}^{-1} \mathrm{~s}^{-1}$ at room temperature. In a next step, the graphene was patterned by standard e-beam lithography, such that source and drain electrodes were defined (channel length $l=(5-10) \mu \mathrm{m}$, width $w=1000 \mu \mathrm{m}$ or $w=400 \mu \mathrm{m}$ ) and fitted with Ti/Au contact pads. P3HT was spin coated and subsequently patterned by an oxygen etch using an aluminum mask such that P3HT covered the source, channel and drain area. For the fullerene samples, 
fullerenes were deposited by thermal evaporation through a PMMA shadow mask (thickness (70-100) nm), and immediately transferred to a cryostat (sample in vacuum). For the latter, care was taken that the freshly prepared fullerene layer was exposed to air less than 5 minutes. The device structure is sketched in Fig. 1.

\section{Results and discussion}

For the analysis of data, we use the standard MOSFET models. ${ }^{18}$ They distinguish two regimes. The first is the linear regime, when the difference between gate and threshold voltage is greater than the drain voltage $\left(V_{\mathrm{GS}}-V_{\mathrm{T}} \gg V_{\mathrm{DS}}\right)$. In this case the source current depends on the drain voltage as

$$
I_{\text {lin }}=\frac{\mu C w}{l} V_{\mathrm{DS}}\left(V_{\mathrm{GS}}-V_{\mathrm{T}}-\frac{V_{\mathrm{DS}}}{2}\right)
$$

with threshold voltage $V_{\mathrm{T}}$ and the gate capacitance $C$ per area. $C$ $=6.9 \mathrm{nF} \mathrm{cm}^{-2}$ was determined from the plate capacitor formula with $\mathrm{SiC}$ as dielectric $(d=1.25 \mu \mathrm{m})$. The validity of this approximation was previously confirmed. ${ }^{16,17}$

In the second regime, when the drain voltage is equal or greater than the difference between gate and threshold voltage $\left(V_{\mathrm{DS}} \geq V_{\mathrm{GS}}-V_{\mathrm{T}}\right)$, the current saturates due to the channel pinchoff and is given by:

$$
I_{\mathrm{sat}}=\frac{\mu C w}{2 l}\left(V_{\mathrm{GS}}-V_{\mathrm{T}}\right)^{2}
$$

Note that eqn (1) and (2) are exemplarily given for n-channel MOSFETs. For p-channel FETs the sign of the voltages has to be changed.

\section{Polythiophene as prototypical p-type semiconductor}

A first and all-important step is to check the electrical contact properties of the graphene-semiconductor interface. For this purpose, we used bottom-gated transistor structures with various channel lengths $l$. At first, we discuss the case of P3HT.

Fig. 2a shows the normalized zero-bias resistance $R_{\operatorname{lin}} \times w$ determined from the linear regime for voltages $V_{\mathrm{DS}}<2 \mathrm{~V}$ as a function of the channel length $l$. For all gate voltages $V_{\mathrm{GS}}$

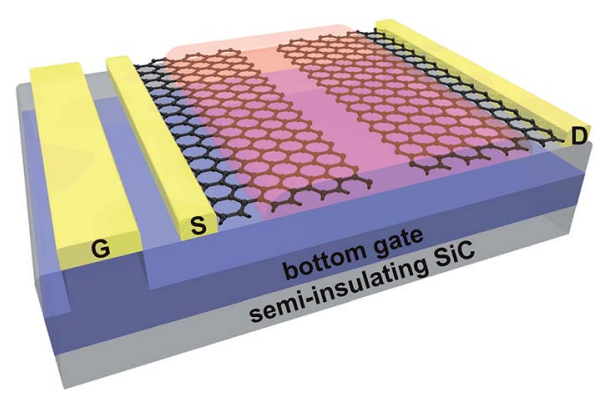

Fig. 1 Schematic of the transistor testbed with epitaxial graphene as source and drain electrode, and an implanted bottom gate. The red area on top represents the semiconductor in the channel. In this work, we used P3HT or fullerene as example.
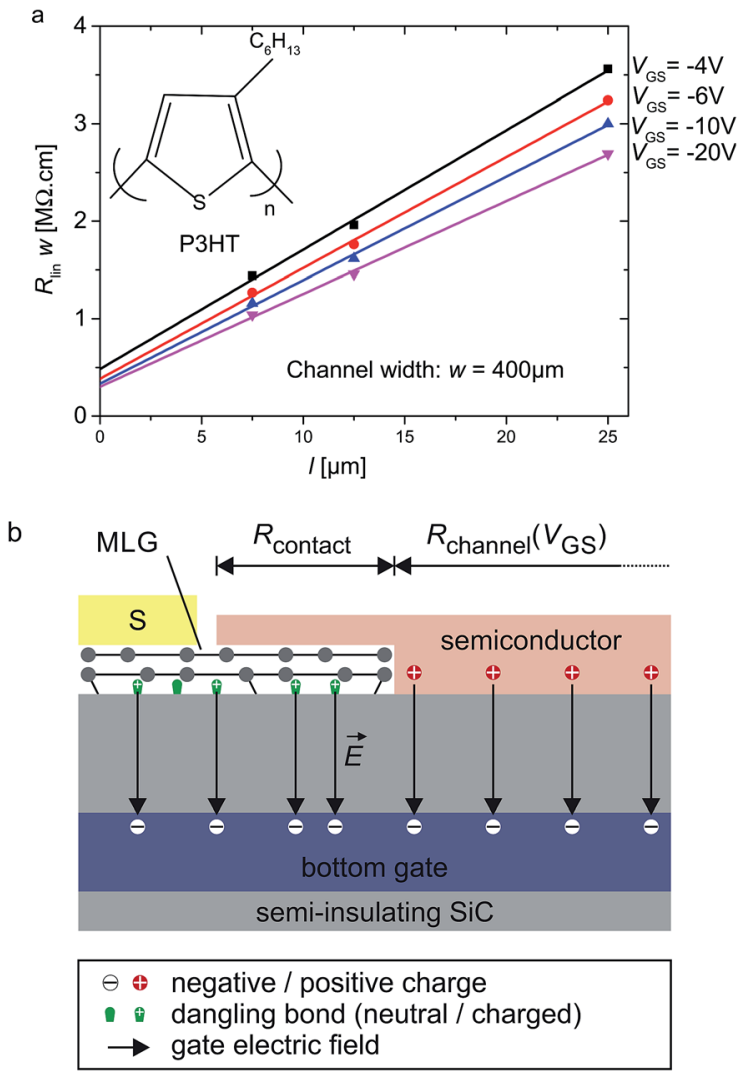

Fig. 2 (a) Normalized zero-bias resistance $R_{\text {lin }} \times w$ measured on three P3HT transistors with different channel length $l$, each evaluated for four gate voltages $V_{G S}$. The contact behaves entirely ohmic. The contact resistance $R_{\text {contact }}$ is extracted using the transfer length method (TLM). (b) Scheme of the contributions to $R_{\text {lin }}=2 R_{\text {contact }}+$ $R_{\text {channel }}\left(V_{\mathrm{GS}}\right)$. The arrows indicate the gate electric field, which influences only $R_{\text {channel }}\left(V_{\mathrm{GS}}\right)$ of the semiconductor in the transistor channel. The graphene/semiconductor contact areas with $R_{\text {contact }}$ are unaffected by the gate due to Fermi level pinning at dangling bond states below graphene.

investigated, a linear dependence is observed. The extrapolation to zero length results in a contact resistance $R_{\text {contact }}=(0.20 \pm$ $0.05) \mathrm{M} \Omega \mathrm{cm}$. This value is almost independent of the channel resistance $R_{\text {channel }}$ of each device, which is parametrically varied by $V_{\mathrm{GS}}$. This is expected as the monolayer graphene/SiC interface screens the gate effect of the bottom gate due to Fermi level pinning, ${ }^{16,17}$ which is enforced by silicon dangling bonds underneath the graphene layer. Note that the P3HT on top of the graphene contact is consequently ungated. $R_{\text {contact }}$ therefore includes the graphene resistance (negligible), the transition from graphene to ungated $\mathrm{P} 3 \mathrm{HT}$ and the resistive pathway in P3HT above the source and drain electrodes (see Fig. 2b). This evaluation scheme cannot be applied to fullerenes, as the zerobias resistance is not linear (see below).

For the given transistor scheme, two problems occurred, which had to be solved: (i) gate electric field screening by surface states and (ii) gate leakage.

Ad (i). In first experiments, we observed almost no gate effect as can be seen in Fig. 3a. This problem was presumably due to surface-near defects in the substrate or interface, which were 
a

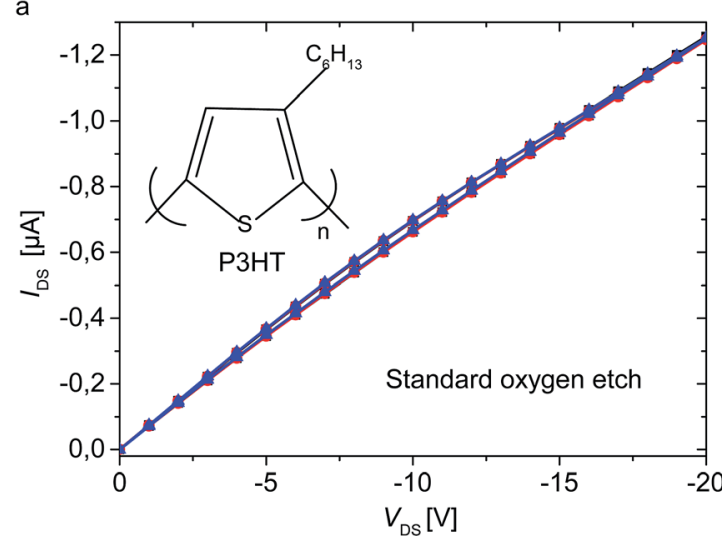

b

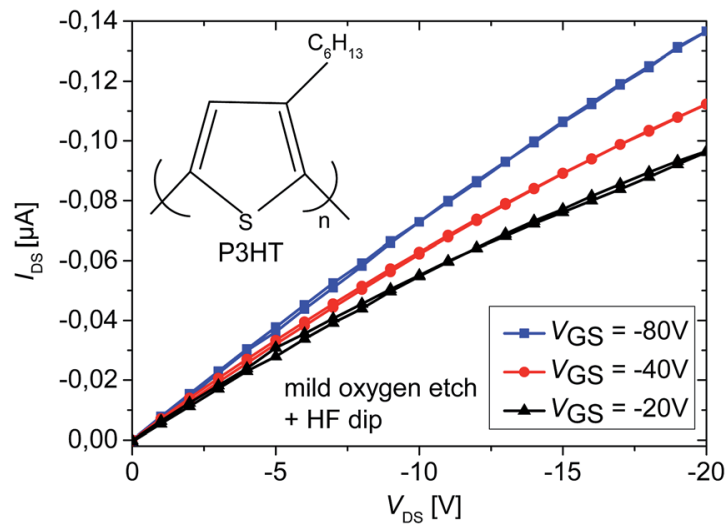

Fig. 3 Output characteristics of P3HT transistors measured in both sweep directions $\left(V_{D S}=0 \rightarrow-20 \mathrm{~V} \rightarrow 0\right.$ ) indicate the sensitivity to the graphene removal process: (a) oxygen plasma with RF power 100 W, 15 $\mathrm{s}$ without subsequent HF dip; almost no gate effect is visible, (b) improved gate effect due to mild conditions (15 W, $1 \mathrm{~min}$ ) and subsequent HF dip. An Al implanted bottom gate was used. The legend refers to both (a) and (b).

generated by the removal of the graphene in the channel area. It turned out that milder conditions (RF power: $15 \mathrm{~W}, 1 \mathrm{~min}$ instead of $100 \mathrm{~W}, 15 \mathrm{~s}$ ) during the oxygen plasma etch as well as a HF dip prior to semiconductor deposition solved the problem (see Fig. 3b). We conclude that the gate effect was spoiled either by plasma-induced surface-near defects in SiC, which are avoided by milder etching conditions, or by the uncontrolled formation of a thin $\mathrm{SiO}_{2}$ layer with high interface trap density.

Ad (ii). Despite our expectation that an aluminum-doped bottom gate is favorable for the creation of holes in p-type materials, ${ }^{17}$ it turned out that for the given experiment nitrogen-doping is advantageous. Under these conditions, the n-type/intrinsic junction is driven in forward direction. This limits the applicable gate voltage to approximately $-20 \mathrm{~V}$. The advantage, however, is that less implantation-induced defects are created in the gate dielectric, which reduces the gate leakage current significantly. In the following, only nitrogen-doped bottom gates are used.

Fig. 4 shows the output and transfer characteristics taken on a P3HT transistor fabricated with optimized processing
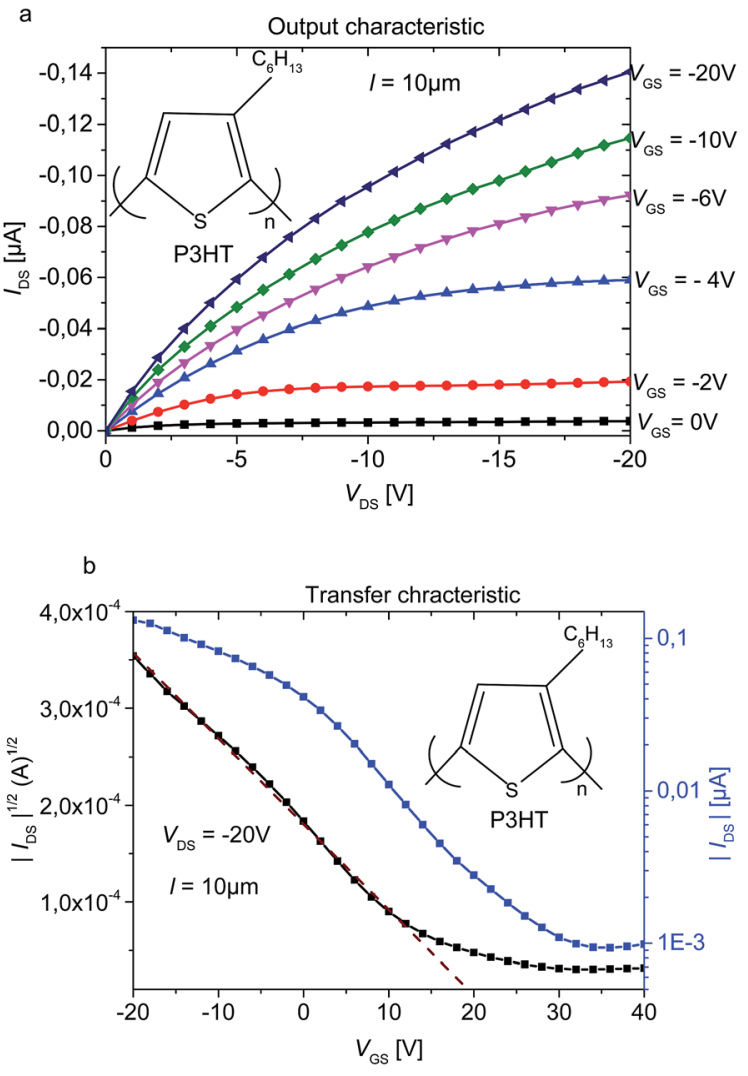

Fig. 4 P3HT transistor: (a) output characteristic and (b) transfer characteristic measured at room temperature. The linear regime, the drain current saturation at higher source-drain voltages of the output characteristic as well as the clear quadratic dependence of $I_{D S}\left(V_{G S}\right)$ allow for accurate determination of transistor parameters according to standard MOSFET models.

conditions. The output characteristic clearly reveals a linear regime for source-drain voltages $V_{\mathrm{DS}}<2 \mathrm{~V}$ and a saturation regime at high $V_{\mathrm{DS}}$ values (dependent on the gate voltage $V_{\mathrm{GS}}$ ). The transfer characteristic measured at $V_{\mathrm{DS}}=-20 \mathrm{~V}$ is depicted in Fig. $4 \mathrm{~b}$. The left scale corresponds to the square root of $I_{\mathrm{DS}}$. The linear curve observed at $V_{\mathrm{GS}}<5 \mathrm{~V}$ proves that the transistor behaves closely to an ideal MOSFET and eqn (2) can be applied for the saturation current.

The on/off ratio of the P3HT transistor is $\approx 10^{2}$. From the transfer characteristic, a threshold voltage $V_{\mathrm{T}}=20 \mathrm{~V}$ is obtained by extrapolation of the $I_{\mathrm{DS}}{ }^{1 / 2}-V_{\mathrm{GS}}$ curve ( $c f$. Fig. 4b). Further, from the maximum slope $\mathrm{d} I_{\mathrm{DS}}{ }^{1 / 2} / \mathrm{d} V_{\mathrm{GS}}$ a field-effect mobility of $\mu_{\mathrm{FE}} \approx 5 \times 10^{-4} \mathrm{~cm}^{2} \mathrm{~V}^{-1} \mathrm{~s}^{-1}$ is extracted, which is a typical value for P3HT. The effective mobility $\mu_{\text {eff }}=5 \times 10^{-4} \mathrm{~cm}^{2} \mathrm{~V}^{-1} \mathrm{~s}^{-1}$ can be calculated from the slope of the linear regime of the output characteristic employing eqn (1) for $V_{\mathrm{GS}}<-5 \mathrm{~V}$, where it is in perfect agreement with $\mu_{\mathrm{FE}}$. We explicitly included the contact resistance $R_{\text {contact }}$ by rescaling of $V_{\mathrm{DS}}$ :

$$
V_{\mathrm{DS}, \text { channel }}=V_{\mathrm{DS} \text {,measured }}-2 R_{\text {contact }} I_{\mathrm{DS}}
$$

This correction is relatively small $(<10 \%)$, which displays the high quality of the all-carbon graphene to P3HT contact. 


\section{Fullerene as prototypical n-type semiconductor}

As an example for an n-type semiconductor, we used a fullerene layer deposited by thermal evaporation. On a first glance, the fullerene/graphene contact seems to be particularly homogeneous, as two pure $\mathrm{sp}^{2}$ carbon materials are in contact. However, when investigating these transistors at room temperature, very poor conduction was observed. The bad conductivity is related to rotational dynamics of the single fullerene molecules. ${ }^{19}$ The conduction improved substantially by three orders of magnitude when cooling down the sample to about $100 \mathrm{~K}$. At this temperature, the rotational dynamics is largely frozen out.

Fig. 5a displays the output characteristics of a fullerene transistor with graphene electrodes at $100 \mathrm{~K}$. As expected for an n-type semiconductor, the source-drain current $I_{\mathrm{DS}}$ increases with increasing gate voltage $V_{\mathrm{GS}}$. Although there is a significant gate effect, a linear regime at small $V_{\mathrm{DS}}$ is missing. Instead, a super-linear increase of $I_{\mathrm{DS}}$ is observed. This indicates that the fullerene/graphene contact is not ohmic, but rather Schottkylike. This observation elucidates how different both carbon allotropes are electronically. Nevertheless, the transistor is functional and reaches on/off ratios of $3 \times 10^{3}$ as can be seen in the transfer characteristic (Fig. 5b). The current $I_{\mathrm{DS}}$ in the saturation regime depends on $V_{\mathrm{GS}}{ }^{2}$ Thus, a field effect mobility according to eqn (2) can be evaluated resulting in $\mu_{\mathrm{FE}}=0.1 \mathrm{~cm}^{2}$
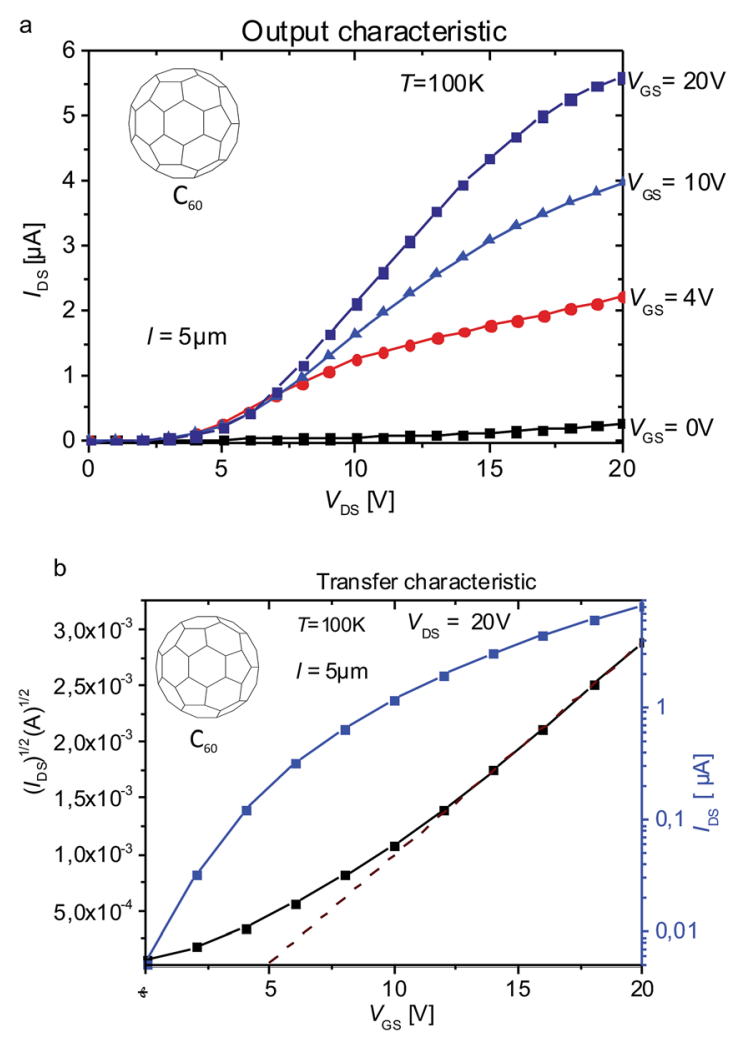

Fig. 5 Fullerene transistor: (a) output characteristic and (b) transfer characteristic measured at $T=100 \mathrm{~K}$. Although the linear regime is missing due to Schottky-like fullerene/graphene contacts, transistor operation with on/off ratio of $3 \times 10^{3}$ is observed.
$\mathrm{V}^{-1} \mathrm{~s}^{-1}$ for $V_{\mathrm{GS}}>10 \mathrm{~V}$. Note that this value is a rough estimate as the contact resistance is essentially unknown and may influence the result significantly.

\section{Conclusions}

Epitaxial graphene with implanted bottom gate in $\mathrm{SiC}(0001)$ can be used as a testbed to investigate the semiconducting fieldeffect transistor operation of new materials under extremely well-controlled conditions. The well-defined work function of epitaxial graphene (independent of applied backgate voltages) and its zero roughness provide reproducible (metallic) source and drain electrodes without any gate effect in the contact area. The zero roughness continues also in the bottom gated transistor channel area at the clean and atomically flat SiC surface with practically no step in between. The screening effect of the SiC/epitaxial graphene interface provides a spatially sharp separation of contact area and channel.

For the p-type semiconductors P3HT, perfect ohmic injection and transistor operation according to standard MOSFET models is observed. This allows an accurate determination of parameters. We expect that this analysis can be extended to many other p-type organic semiconductors electronically similar to P3HT. The testbed can be used also for fullerenes as an example for n-type semiconductors, where transistor operation was observed. The obvious Schottky-like contacts indicate work function mismatch. Consequently, a linear regime in the output characteristic is missing and the determination of parameters is less meaningful.

\section{Acknowledgements}

The work was carried out in the framework of the collaborative research centre SFB 953, funded by DFG. We acknowledge fruitful discussions with Petra Rudolf, Gebhard Matt, and Vojislav Krstic.

\section{Notes and references}

1 A. K. Geim and K. S. Novoselov, Nat. Mater., 2007, 6, 183-191. 2 Y.-M. Lin, A. Valdes-Garcia, S.-J. Han, D. B. Farmer, I. Meric, Y. Sun, Y. Wu, C. Dimitrakopoulos, A. Grill, P. Avouris and K. A. Jenkins, Science, 2011, 332, 1294-1297.

3 Y. M. Lin, C. Dimitrakopoulos, K. A. Jenkins, D. B. Farmer, H. Y. Chiu, A. Grill and P. Avouris, Science, 2010, 327, 662.

4 F. Schwierz, Nat. Nanotechnol., 2011, 5, 487-496.

5 M. Choe, B. H. Lee, G. Jo, J. Park, W. Park, S. Lee, W. K. Hong, M. J. Seong, Y. H. Kahng, K. Lee and T. Lee, Org. Electron., 2010, 11, 1864-1869.

6 S. Lee, S. J. Kang, G. Jo, M. Choe, W. Park, J. Yoon, T. Kwon, Y. H. Kahng, D. Y. Kim, B. H. Lee and T. Lee, Appl. Phys. Lett., 2011, 99, 083306.

7 S. Lee, G. Jo, S. J. Kang, G. Wang, M. Choe, W. Park, D. Y. Kim, Y. H. Kahng and T. Lee, Adv. Mater., 2011, 23, 100-105. 
8 L. Sangchul, Y. Jun-Seok, J. Yongsung, C. Chunhum, K. Dong-Yu, N. Seok-In, L. Byoung Hun and L. Takhee, Nanotechnology, 2012, 23, 344013.

9 W. H. Lee, J. Park, S. H. Sim, S. Lim, K. S. Kim, B. H. Hong and K. Cho, J. Am. Chem. Soc., 2011, 133, 4447-4454.

10 W. H. Lee, J. Park, S. H. Sim, S. B. Jo, K. S. Kim, B. H. Hong and K. Cho, Adv. Mater., 2011, 23, 1752-1756.

11 S. Hertel, D. Waldmann, J. Jobst, A. Albert, M. Albrecht, S. Reshanov, A. Schoner, M. Krieger and H. B. Weber, Nat. Commun., 2012, 3, 957.

12 M. Brinkmann, J. Polym. Sci., Part B: Polym. Phys., 2011, 49, 1218-1233.

13 X. H. Zhang and B. Kippelen, Appl. Phys. Lett., 2008, 93, 133305.

14 T. D. Anthopoulos, B. Singh, N. Marjanovic, N. S. Sariciftci, A. Montaigne Ramil, H. Sitter, M. Cölle and D. M. de Leeuw, Appl. Phys. Lett., 2006, 89, 213504.
15 K. V. Emtsev, A. Bostwick, K. Horn, J. Jobst, G. L. Kellogg, L. Ley, J. L. McChesney, T. Ohta, S. A. Reshanov, J. Rohrl, E. Rotenberg, A. K. Schmid, D. Waldmann, H. B. Weber and T. Seyller, Nat. Mater., 2009, 8, 203-207.

16 D. Waldmann, J. Jobst, F. Speck, T. Seyller, M. Krieger and H. B. Weber, Nat. Mater., 2011, 10, 357-360.

17 D. Waldmann, J. Jobst, F. Fromm, F. Speck, T. Seyller, M. Krieger and H. B. Weber, J. Phys. D: Appl. Phys., 2012, 45, 154006.

18 S. M. Sze and K. K. Ng, Physics of Semiconductor Devices, Wiley Interscience, 2007.

19 N. M. Nemes, M. Garcia-Hernandez, G. Bortel, E. Kovats, B. J. Nagy, I. Jalsovszky and S. Pekker, J. Phys. Chem. B, 2009, 113, 2042-2049. 